\title{
Linking Partial Dynamical Symmetry to Nuclear Energy Density Functionals
}

\author{
A. Leviatan ${ }^{1}$, N. Gavrielov ${ }^{1}$, K. Nomura ${ }^{2}$ \\ ${ }^{1}$ Racah Institute of Physics, The Hebrew University, Jerusalem 91904, Israel \\ ${ }^{2}$ Department of Physics, Faculty of Science, University of Zagreb, \\ HR-10000 Zagreb, Croatia
}

Received 15 November 2021

doi: https://doi.org/10.55318/bgjp.2022.49.1.067

\begin{abstract}
We use self-consistent mean-field methods in combination with the interacting boson model (IBM) of nuclei, to establish a linkage between universal energy density functionals (EDFs) and partial dynamical symmetry (PDS). An application to ${ }^{168} \mathrm{Er}$ shows that IBM Hamiltonians derived microscopically from known nonrelativistic and relativistic EDFs in this region, conform with SU(3)-PDS.
\end{abstract}

KEY WORDS: Nuclear energy density functionals, partial dynamical symmetry, $\mathrm{SU}(3)$ structure in ${ }^{168} \mathrm{Er}$.

\section{Introduction}

The concept of dynamical symmetry (DS) is by now widely recognized to play a key role in the structure of nuclei. Its basic paradigm is to write the Hamiltonian of the system in terms of the Casimir operators of a chain of nested algebras, $G_{\text {dyn }} \supset G_{1} \supset G_{2} \supset \cdots \supset G_{\text {sym }}$, where $G_{\text {dyn }}$ is the dynamical (spectrum generating) algebra of the system such that operators of all physical observables can be written in terms of its generators and $G_{\text {sym }}$ is the symmetry algebra [1]. A dynamical symmetry is characterized by complete solvability for all states in terms of quantum numbers, which are the labels of irreducible representations (irreps) of the algebras in the chain.

A notable example of such algebraic construction is the interacting boson model (IBM) [2], which describes low-lying quadrupole collective states in nuclei in terms of $N$ monopole $(s)$ and quadrupole $(d)$ bosons. In this case, $G_{\text {dyn }}=\mathrm{U}(6)$ and $G_{\text {sym }}=\mathrm{SO}(3)$. The model accommodates several DS chains with leading subalgebra $G_{1}=\mathrm{U}(5), \mathrm{SU}(3)$ and $\mathrm{SO}(6)$, whose spectra resemble known paradigms of nuclear collective structure: spherical vibrator, axially-deformed rotor and $\gamma$-soft deformed rotor, respectively. 
Geometry is introduced in the IBM through an energy surface,

$$
E_{\mathrm{IBM}}(\tilde{\beta}, \gamma)=\langle\tilde{\beta}, \gamma ; N|\hat{H}| \tilde{\beta}, \gamma ; N\rangle
$$

defined by the expectation value of the Hamiltonian in a coherent state $[3,4]$,

$$
\begin{aligned}
& |\tilde{\beta}, \gamma ; N\rangle=(N !)^{-1 / 2}\left[b_{c}^{\dagger}(\tilde{\beta}, \gamma)\right]^{N}|0\rangle \\
& b_{c}^{\dagger}(\tilde{\beta}, \gamma)=\left(1+\tilde{\beta}^{2}\right)^{-1 / 2}\left[\tilde{\beta} \cos \gamma d_{0}^{\dagger}+\tilde{\beta} \sin \gamma\left(d_{2}^{\dagger}+d_{-2}^{\dagger}\right) / \sqrt{2}+s^{\dagger}\right]
\end{aligned}
$$

Here $(\tilde{\beta}, \gamma)$ are quadrupole shape parameters in the IBM, whose values, $\left(\beta_{0}, \gamma_{0}\right)$, at the global minimum of $E_{\mathrm{IBM}}(\tilde{\beta}, \gamma)$ define the equilibrium shape for a given Hamiltonian. The shape can be spherical $\left(\beta_{0}=0\right)$ or deformed $\left(\beta_{0}>0\right)$ with $\gamma_{0}=0^{\circ}$ (prolate), $\gamma_{0}=60^{\circ}$ (oblate), $0^{\circ}<\gamma_{0}<60^{\circ}$ (triaxial) or $\gamma$ independent. The equilibrium deformations associated with the DS limits, conform with their geometric interpretation, and are given by $\beta_{0}=0$ for $\mathrm{U}(5)$, $\left(\beta_{0}=\sqrt{2}, \gamma_{0}=0^{\circ}\right)$ for $\mathrm{SU}(3)$ and $\left(\beta_{0}=1, \gamma_{0}\right.$ arbitrary) for $\mathrm{SO}(6)$. The coherent state $\left|\beta_{0}, \gamma_{0} ; N\right\rangle$ (2), with the equilibrium deformations, serves as the intrinsic state for the ground band.

The merits of a DS, with its analytic and geometric attributes, are self evident. However, in the majority of nuclei, an exact DS rarely occurs and one is compelled to break it. More often some states obey the patterns required by the symmetry, but others do not. The need to address such situations, but still preserve important symmetry remnants, has motivated the introduction of partial dynamical symmetry (PDS) [5,6]. The essential idea is to relax the stringent conditions imposed by an exact DS so that solvability and/or good quantum numbers are retained by only a subset of states. Detailed studies in the IBM framework, have shown that PDSs account quite well for a wealth of spectroscopic data in various types of nuclei [5-11] and are relevant to related quantum phase transitions and shape-coexistence [12-16]. In all these phenomenological studies, an Hamiltonian with a prescribed PDS is introduced, its parameters are determined from a fit to the spectra, and the PDS predictions (which are often parameter-free) are compared with the available empirical energies and transition rates. In the present contribution, we show that the PDS notion is robust and founded on microscopic grounds [17].

PDSs do not arise from invariance properties of the Hamiltonian, hence can be referred to as emergent symmetries. The role of an emergent $\operatorname{Sp}(3, \mathrm{R}) \mathrm{DS}$ has been recently demonstrated within ab-initio calculations of light nuclei $[18,19]$. Here we focus on heavy nuclei, and present an efficient procedure to uncover the microscopic origin of PDS by linking it to universal nuclear energy density functionals. We apply the procedure to ${ }^{168} \mathrm{Er}$, in which the SU(3)-PDS was previously recognized on phenomenological grounds [6-8]. 


\section{SU(3) PDS: A Phenomenological Approach}

The SU(3) dynamical symmetry limit and basis states correspond to the chain,

$$
\mathrm{U}(6) \supset \mathrm{SU}(3) \supset \mathrm{SO}(3) \quad|[N](\lambda, \mu) K L\rangle .
$$

The SU(3)-DS Hamiltonian involves the Casimir operators, $\hat{C}_{\mathrm{G}}$, of the algebras in the chain. The spectrum consists of SU(3) multiplets with the states $|[N](\lambda, \mu) K L\rangle$ specified by the total boson number $N$, the $\operatorname{SU}(3)$ irrep $(\lambda, \mu)$, the angular momentum $L$, and the label $K$ which corresponds to the projection of the angular momentum on the symmetry axis. The lowest multiplets have $(\lambda, \mu)=(2 N, 0)$ which contains the ground band $g(K=0)$, and $(\lambda, \mu)=(2 N-4,2)$ which contains both the $\beta(K=0)$ and $\gamma(K=2)$ bands.

Following the general algorithm [5,10,20], the SU(3)-PDS Hamiltonian is constructed to be [6],

$$
\hat{H}_{\mathrm{PDS}}=h_{0} P_{0}^{\dagger} P_{0}+h_{2} P_{2}^{\dagger} \cdot \tilde{P}_{2}+\rho \hat{L} \cdot \hat{L} .
$$

Here $P_{0}^{\dagger}=d^{\dagger} \cdot d^{\dagger}-2\left(s^{\dagger}\right)^{2}, P_{2 m}^{\dagger}=2 d_{m}^{\dagger} s^{\dagger}+\sqrt{7}\left(d^{\dagger} d^{\dagger}\right)_{m}^{(2)}, \tilde{P}_{2 m}=(-1)^{m} P_{2,-m}$, $\hat{L}$ the angular momentum operator and standard notation of angular momentum coupling is used. For $h_{0}=h_{2}, \hat{H}_{\mathrm{PDS}}$ reduces to the SU(3)-DS Hamiltonian $\hat{H}_{\mathrm{DS}}=h_{2}\left[-\hat{C}_{\mathrm{SU}(3)}+2 \hat{N}(2 \hat{N}+3)\right]+\rho \hat{C}_{\mathrm{SO}(3)}$. For $h_{0} \neq h_{2}, \hat{H}_{\mathrm{PDS}}$ is no longer diagonal in the SU(3)-DS chain (3), but still has a subset of eigenstates with good SU(3) symmetry. This comes about because $P_{0}$ and $P_{2 m}$ annihilate the states $|[N](2 N, 0) K=0, L\rangle$ comprising the ground band $g(K=0)$ and $P_{0}$ annihilates also the states $|[N](2 N-4 k, 2 k) K=2 k, L\rangle$ comprising the $\gamma^{k}(K=2 k)$ bands. In particular, the ground and gamma bands remain solvable with good SU(3) quantum numbers, $(\lambda, \mu)=(2 N, 0)$ and $(2 N-4,2)$, and energies

$$
\begin{aligned}
& g(K=0): E=\rho L(L+1), \\
& \gamma(K=2): E=h_{2} 6(2 N-1)+\rho L(L+1),
\end{aligned}
$$

while the $\beta(K=0)$ band is mixed.

In a phenomenological approach, the parameters of $\hat{H}_{\mathrm{PDS}}$ are determined from a fit to experimental energies; $h_{2}$ and $\rho$ from $E\left(2_{1}\right)$ and $E\left(2_{2}\right)$, using Eq. (5), and $h_{0}$ from $E\left(0_{2}\right)$. As shown in Figure 1, a PDS calculation with parameters indicated in the caption, provides a good description for the lowest bands in ${ }^{168} \mathrm{Er}$. [6]. The ground and gamma are pure SU(3) bands, but the beta band is found to contain $13 \%$ admixtures into the dominant $(2 N-4,2)$ irrep [7]. Since the wave functions of the solvable states are known, it is possible to obtain analytic expressions for matrix elements of observables between them. The E2 operator can be transcribed as $\hat{T}(E 2)=\alpha Q^{(2)}+\theta\left(d^{\dagger} s+s^{\dagger} \tilde{d}\right)$, with $Q^{(2)}=$ $d^{\dagger} s+s^{\dagger} \tilde{d}-\frac{\sqrt{7}}{2}\left(d^{\dagger} \tilde{d}\right)^{(2)}$, an SU(3) generator. Since the solvable ground and gamma bands reside in different $\mathrm{SU}(3)$ irreps, $Q^{(2)}$ cannot connect them and, 

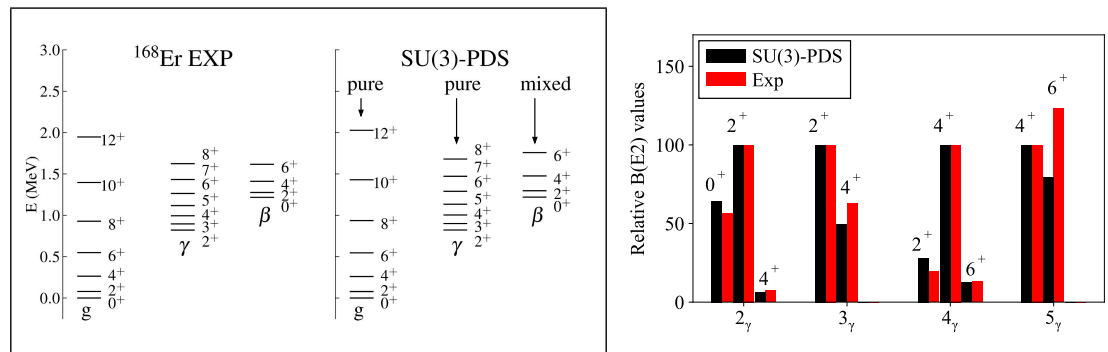

Figure 1. (Color online) Left panels: observed spectrum of ${ }^{168} \mathrm{Er}$ compared to an SU(3)PDS calculation employing $\hat{H}_{\mathrm{PDS}}$ of Eq. (4) with $h_{0}=8, h_{2}=4, \rho=13 \mathrm{keV}$ and $N=16$, for which the ground and $\gamma$ bands are pure while the $\beta$ band is mixed, with respect to SU(3). Right panel: comparison of the PDS parameter-free predictions with the data on relative $B\left(E 2 ; L_{\gamma} \rightarrow L\right)$ values for $\gamma \rightarrow g E 2$ transitions in ${ }^{168}$ Er. Adapted from $[6,8]$.

consequently, $B(E 2)$ ratios for $\gamma \rightarrow g$ transitions do no depend on the $E 2$ parameters $(\alpha, \theta)$ nor on parameters of the PDS Hamiltonian (4). Overall, as shown in the right panel of Figure 1, these parameter-free predictions of SU(3)PDS account well for the data in ${ }^{168} \operatorname{Er}[6,8]$.

$\hat{H}_{\mathrm{PDS}}$ in Eq. (4) decomposes naturally into intrinsic and collective parts. The former, consisting of the $h_{0}$ and $h_{2}$ terms, determines the energy surface (1) and band-structure, while the latter, consisting of the $\rho$ term, determines the in-band rotational splitting. Such a resolution is valid also for the general IBM Hamiltonian describing the dynamics of a prolate-deformed shape, which reads [21],

$$
\hat{H}=h_{0} P_{0}^{\dagger}\left(\beta_{0}\right) P_{0}\left(\beta_{0}\right)+h_{2} P_{2}^{\dagger}\left(\beta_{0}\right) \cdot \tilde{P}_{2}\left(\beta_{0}\right)+\rho \hat{L} \cdot \hat{L} .
$$

Here $P_{0}^{\dagger}\left(\beta_{0}\right)=d^{\dagger} \cdot d^{\dagger}-\beta_{0}^{2}\left(s^{\dagger}\right)^{2}$ and $P_{2 m}^{\dagger}\left(\beta_{0}\right)=\beta_{0} \sqrt{2} d_{m}^{\dagger} s^{\dagger}+\sqrt{7}\left(d^{\dagger} d^{\dagger}\right)_{m}^{(2)}$. Its energy surface, obtained from Eq. (1), is given by

$$
\begin{aligned}
E_{\mathrm{IBM}}(\tilde{\beta}, \gamma)=N(N-1) & \left(1+\tilde{\beta}^{2}\right)^{-2}\left[h_{0}\left(\tilde{\beta}^{2}-\beta_{0}^{2}\right)^{2}\right. \\
& \left.+2 h_{2} \tilde{\beta}^{2}\left(\tilde{\beta}^{2}-2 \beta_{0} \tilde{\beta} \cos 3 \gamma+\beta_{0}^{2}\right)\right] .
\end{aligned}
$$

For $h_{0}, h_{2} \geqslant 0$, the surface has a global minimum at $\left(\tilde{\beta}=\beta_{0}>0, \gamma=0^{\circ}\right)$, corresponding to a prolate-deformed equilibrium shape. The contribution of the rotational $\rho$-term to the energy surface is $1 / N$ suppressed, hence negligible. $P_{0}\left(\beta_{0}\right)$ and $P_{2 m}\left(\beta_{0}\right)$ annihilate the states with angular momentum $L$ projected from the intrinsic state $\left|\beta_{0}, \gamma_{0}=0 ; N\right\rangle$ (2). The Hamiltonian $\hat{H}$ of Eq. (6), reduces to $\hat{H}_{\mathrm{PDS}}$ of Eq. (4), when the following conditions are met,

$$
\mathrm{SU}(3) \operatorname{PDS}: \quad h_{0} \neq h_{2}, \beta_{0}=\sqrt{2} .
$$

In what follows, we show that IBM Hamiltonians derived from microscopic considerations for ${ }^{168} \mathrm{Er}$, exhibit spectral properties of SU(3)-PDS. 


\section{SCMF to IBM Mapping}

The nuclear energy density functional (EDF) framework allows for a reliable quantitative prediction of ground-state properties and collective excitations of nuclei over the entire region of the nuclear chart. Its basic implementation is in self-consistent mean-field (SCMF) methods, in which an EDF is constructed as a functional of one-body nucleon density matrices that correspond to a single product state. Pairing correlations are taken into account by a choice of pairing force. In the present contribution, we consider both nonrelativistic [22, 23] and relativistic $[24,25]$ EDFs, so as to ensure the robustness of the results.

The starting point is a set of constrained SCMF calculations of an energy surface [26]. The constraints refer to those for mass quadrupole moments, which are associated with the deformation parameters $\beta$ and $\gamma$ [27]. For the nonrelativistic SCMF calculations, we employ the the Hartree-Fock plus BCS model [28, 29] with two parameterizations of the Skyrme EDF [26] and a density-dependent delta force with strength $V_{0}$. Specifically, the SLy4 [30] parameterization with pairing strengths $V_{0}=1000$ and $1250 \mathrm{MeVfm}^{3}$, and the SkP [31] parameterization with $V_{0}=800$ and $1000 \mathrm{MeVfm}^{3}$. A smooth cut-off of $5 \mathrm{MeV}$ below and above the Fermi surface is invoked for these zero-range pairing forces [31]. For the relativistic SCMF calculations, we employ the relativistic Hartree-Bogoliubov model [24,32] with two types of EDFs. Specifically, the density-dependent point-coupling (DD-PC1) [33] and meson-exchange (DDME2) [34] functionals, both with a separable pairing force of finite range [35] and strengths $V_{0}=728$ and $837 \mathrm{MeVfm}^{3}$, resembling a finite-range Gogny interaction D1S.

The calculated SCMF energy surfaces $E_{\mathrm{SCMF}}(\beta, \gamma)$ for ${ }^{168} \mathrm{Er}$, based on the above nonrelativistic and relativistic EDFs, are displayed on the first and third columns of Figure 2, respectively. As seen, all adopted EDFs lead to energy surfaces accommodating a pronounced prolate-deformed global minimum at $\left(\beta \approx 0.35, \gamma=0^{\circ}\right)$. The minimum tends to be less steep, in both the $\beta$ and $\gamma$ directions, for larger pairing strengths. This is anticipated since pairing correlations favor a more spherical shape.

From the ensemble of Hamiltonians given in Eq. (6), the IBM Hamiltonian appropriate for ${ }^{168} \mathrm{Er}$ is derived by the procedure developed in [36-38]. The parameters $\left\{h_{0}, h_{2}, \beta_{0}\right\}$ are determined by mapping the microscopic energy surface $E_{\mathrm{SCMF}}(\beta, \gamma)$, obtained for a given $\mathrm{EDF}$, onto the corresponding IBM surface $E_{\mathrm{IBM}}(\beta, \gamma)$ of Eq. (7). The condition,

$$
E_{\mathrm{SCMF}}(\beta, \gamma) \approx E_{\mathrm{IBM}}(\beta, \gamma)
$$

is imposed to ensure similar topology in the neighborhood of the global minimum. (The two surfaces are expressed in terms of $\beta$, since the IBM and SCMF deformations are related by $\tilde{\beta}=C \beta$, where the constant $C$ is determined by the mapping). $N$ is fixed by the usual boson counting, from the number of valence 

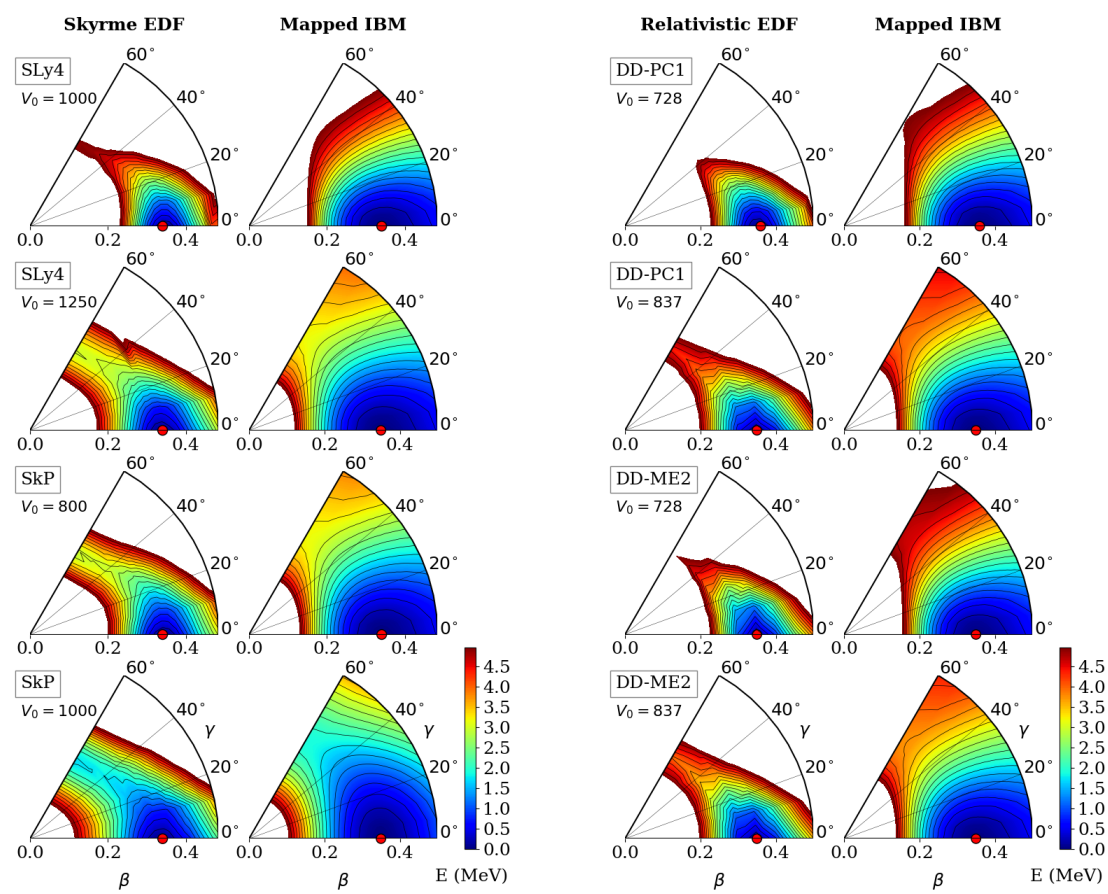

Figure 2. (Color online) SCMF energy surfaces in the $\beta-\gamma$ plane for ${ }^{168} \mathrm{Er}$, based on the nonrelativistic Skyrme SLy4 and SkP EDFs (first column) and the relativistic DD-PC1 and DD-ME2 EDFs (third column) with different values of pairing strengths $V_{0}$ in units of $\mathrm{MeVfm}^{3}$. The corresponding mapped IBM energy surfaces are plotted on the second and fourth columns. Contour spacing is $0.25 \mathrm{MeV}$, and the global minimum is indicated by a solid circle. Adapted from [17].

nucleon pairs counted from the nearest closed shell. The parameter $\rho$, Eq. (6), is obtained by equating the cranking moment of inertia in the IBM to the ThoulessValatin value [39], the procedure discussed in detail in [38]. The mapped IBM energy surfaces, based on the nonrelativistic and relativistic EDFs, are shown on the second and fourth columns of Figure 2, respectively. One clearly sees that the IBM and microscopic surfaces share common essential features near and up to a few $\mathrm{MeV}$ above the global minimum. In what follows, we examine to what extent the derived EDF-based IBM Hamiltonians fulfill the conditions (8) for SU(3)-PDS.

\section{SU(3) PDS: An EDF-Based Approach}

The parameters of the Hamiltonian $\hat{H}$, Eq. (6), derived microscopically from various EDFs, are given in Table 1, along with the parameters of $\hat{H}_{\mathrm{PDS}}$, Eq. (4), 
obtained from a fit to ${ }^{168} \operatorname{Er}$ [6]. As discussed in Section 2, in the latter phenomenological calculation, SU(3)-PDS was pre-assumed, hence condition (8) is satisfied with $\beta_{0}=\sqrt{2}$ and $h_{0} / h_{2}=2$. In comparison, in most SCMF calculations, $1.9<h_{0} / h_{2}<2.8$, consistent with values obtained in global IBM fits in the rare-earth region [7]. The derived values of $\beta_{0}$ are close or slightly larger than the SU(3)-PDS value $\left(\beta_{0}=\sqrt{2} \approx 1.41\right)$. A notable exception are the parameters derived from the SkP EDF with pairing strength $V_{0}=1000 \mathrm{MeVfm}^{3}$, which exhibit pronounced large ratio $h_{0} / h_{2}=6.95$ and small $\beta_{0}=0.99$. This is a consequence of the fact that the corresponding SCMF energy surface for this case, shown in Figure 2, is peculiarly soft in the $\gamma$ deformation, with a shallow local minimum on the oblate side. For any chosen EDF, a larger pairing strength results in a larger (smaller) value for $h_{0} / h_{2}\left(\beta_{0}\right)$.

Excitation spectra appropriate for ${ }^{168} \mathrm{Er}$ are obtained for each EDF by diagonalizing the Hamiltonian (6), using the parameters in Table 1 and $N=16$. Typical spectra resulting from representative nonrelativistic and relativistic EDFs are displayed in Figure 3. They satisfactorily conform with the calculated SU(3)PDS spectrum which, in turn, agrees with experimental spectrum. The bandhead energies, $E\left(2_{2}\right)$ and $E\left(0_{2}\right)$ for the $\gamma$ and $\beta$ bands, and their ratios for the different cases, are listed in Table 1. In general, the description for the ground and $\gamma$ bands is stable with respect to different choices of EDFs. The description of the $\beta$-band is more case-sensitive and all EDFs place $E\left(0_{2}\right)$ above the empirical and SU(3)-PDS values. The following observations are in order. (i) The relativistic EDFs generally result in higher $\beta$-band energies than the Skyrme EDFs. (ii) The increase of the pairing strength $\left(V_{0}\right)$ systematically decreases the $\beta$-band energies. (iii) The SkP EDF with $V_{0}=1000 \mathrm{MeVfm}^{3}$, is the only case where both $E\left(2_{2}\right)$ and $E\left(0_{2}\right)$ are placed below the SU(3)-PDS and empirical values.

Table 1. Parameters $h_{0}, h_{2}, \rho$ (in $\mathrm{keV}$ ) and $\beta_{0}$, of the Hamiltonian (6) obtained from SCMF calculations based on nonrelativistic Skyrme SLy4 and SkP EDFs, and relativistic DD-PC1 and DD-ME2 EDFs, with pairing strengths $V_{0}$ (in $\mathrm{MeV} \mathrm{fm}^{3}$ ). The corresponding parameters for SU(3)-PDS [6], are also shown. $E\left(2_{2}\right)$ and $E\left(0_{2}\right)$ are the calculated bandhead energies (in $\mathrm{keV}$ ) for the $\gamma$ and $\beta$ bands and $R=\frac{E\left(0_{2}\right)}{E\left(2_{2}\right)}$. For ${ }^{168} \mathrm{Er}$, $E\left(2_{2}\right)=821, E\left(0_{2}\right)=1217$ (in keV) and $R=1.48$ [40]. Adapted from [17].

\begin{tabular}{lccccc|ccc}
\hline \hline EDF & $V_{0}$ & $h_{0}$ & $h_{2}$ & $\rho$ & $\beta_{0}$ & $E\left(2_{2}\right)$ & $E\left(0_{2}\right)$ & $R$ \\
\hline SLy4 & 1000 & 10 & 5.3 & 11.8 & 1.59 & 1132 & 1911 & 1.68 \\
& 1250 & 10.4 & 4.0 & 12.3 & 1.39 & 809 & 1334 & 1.65 \\
SkP & 800 & 10.5 & 3.7 & 12.6 & 1.45 & 776 & 1306 & 1.68 \\
& 1000 & 30.6 & 4.4 & 12.2 & 0.99 & 672 & 1087 & 1.62 \\
DD-PC1 & 728 & 10.5 & 5.1 & 11.74 & 1.59 & 1092 & 1889 & 1.73 \\
& 837 & 9.8 & 4.4 & 11.73 & 1.51 & 925 & 1564 & 1.69 \\
DD-ME2 & 728 & 10.4 & 4.8 & 11.74 & 1.59 & 1032 & 1794 & 1.74 \\
& 837 & 9.9 & 4.2 & 11.73 & 1.50 & 883 & 1499 & 1.70 \\
\hline SU(3)-PDS & 8.0 & 4.0 & 13.0 & $\sqrt{2}$ & 822 & 1220 & 1.48 \\
\hline \hline
\end{tabular}



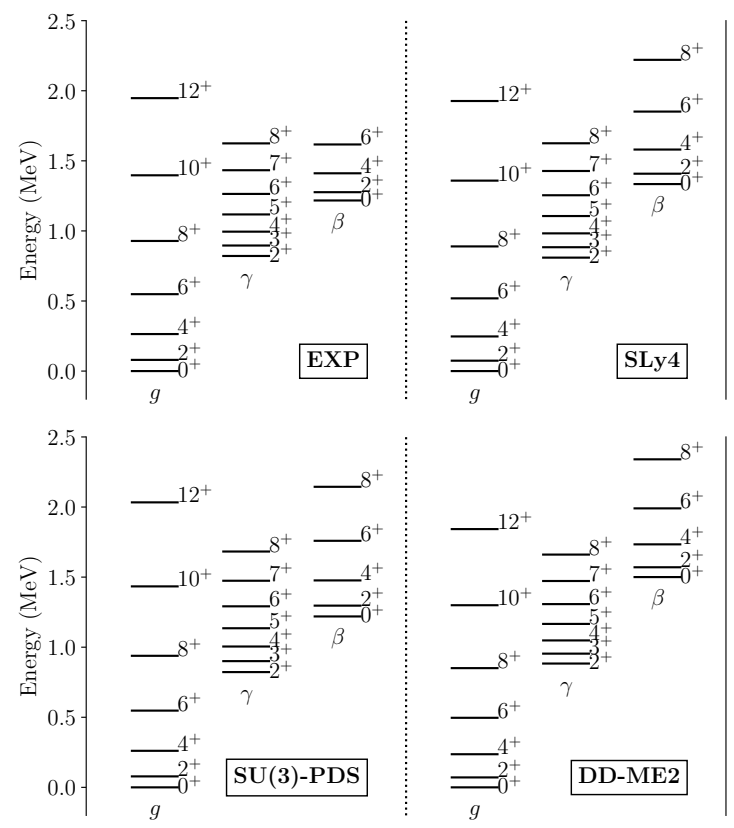

Figure 3. Experimental [40] (EXP) and SU(3)-PDS [6] spectra for ${ }^{168} \mathrm{Er}$, compared with the spectra resulting from EDF-based IBM calculations for the Skyrme SLy4 EDF with pairing strength $V_{0}=1250 \mathrm{MeVfm}^{3}$, and for the relativistic EDF DD-ME2 with $V_{0}=837 \mathrm{MeVfm}^{3}$. Adapted from [17].

Analysis of wave functions is a more sensitive measure to quantify the similarities and differences in structure between the EDF-based IBM Hamiltonians and SU(3)-PDS. Figure 4 shows the $\operatorname{SU}(3)(\lambda, \mu)$-decomposition for member states of the lowest bands in ${ }^{168}$ Er. For SU(3)-PDS, the ground and $\gamma$ bands are pure with $\mathrm{SU}(3)$ character $(2 N, 0)$ and $(2 N-4,2)$, respectively, whereas the $\beta$ band contains a mixture of irreps: $(2 N-4,2) 87.5 \%,(2 N-6,0) 9.6 \%$, and $(2 N-8,4) 2.9 \%$, with $N=16$. Remarkably, for all nonrelativistic and relativistic EDFs considered (except SkP with pairing strength $V_{0}=1000 \mathrm{MeVfm}^{3}$ ), the mapped IBM Hamiltonians reproduce very well the SU(3)-PDS prediction of SU(3)-purity for the ground and $\gamma$ bands, with probability larger than $95 \%$. This clearly demonstrates the robustness of the PDS notion and its microscopic roots. The structure of the $\beta$ band is more sensitive to the choice of EDF. Its $\mathrm{SU}(3)$ mixing is governed by the values of the parameters $\beta_{0}$ and ratio $h_{0} / h_{2}$ which, in turn, reflect the different topology of the corresponding SCMF surfaces. Although the dominance of the $(2 N-4,2),(2 N-6,0)$, and $(2 N-8,4)$ irreps in the $\beta$ band is generally observed in all cases, their relative weights differ from those of SU(3)-PDS. This may indicate that additional degrees of freedom not included in the IBM (e.g., quasi particles) contribute to the structure of the $K=0_{2}$ band in ${ }^{168} \mathrm{Er}$. Again, the situation is different for the EDF SkP with 
Linking Partial Dynamical Symmetry to Nuclear Energy Density Functionals

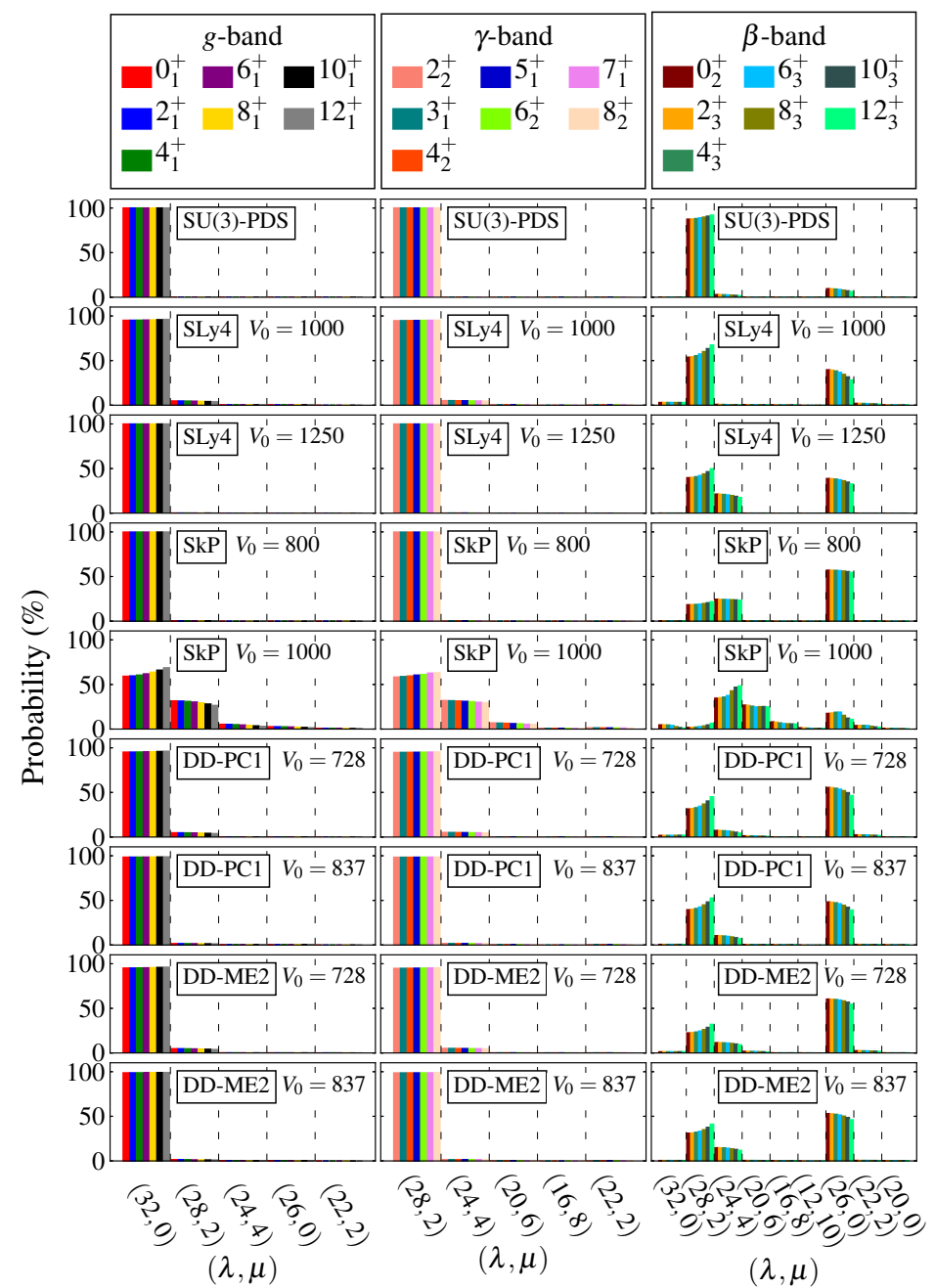

Figure 4. (Color online) $\mathrm{SU}(3)(\lambda, \mu)$-decomposition of states in the ground $(g), \gamma$ and $\beta$ bands, for the SU(3)-PDS and various EDF-based calculations. Shown are probabilities larger than $0.5 \%$. The histograms shown from left-to-right for each band, correspond to the $L_{i}$ states listed in the upper panels in the order top-to-bottom left-to-right. Adapted from [17].

$V_{0}=1000 \mathrm{MeVfm}^{3}$ for which the SU(3) decomposition exhibits large fragmentation. From all the EDFs considered, the SLy4 and SkP with $V_{0}=1250$ and 800 $\mathrm{MeVfm}^{3}$, respectively, appear to yield spectral properties which are closest to the SU(3)-PDS predictions for ${ }^{168} \mathrm{Er}$ (SU(3) purity for the ground and $\gamma$ bands with probability $99.8 \%$ ). 


\section{Conclusions and Outlook}

We have shown that the occurrence of partial dynamical symmetry (PDS) in nuclei can be justified from a microscopic point of view. By employing the constrained mean-field methods with choices of the universal energy density functionals and pairing interactions, in combination with symmetry analysis of the wave functions of the mapped IBM Hamiltonians, we arrived at an efficient procedure to test and explain the emergence of PDS in nuclei. An application to

${ }^{168} \mathrm{Er}$, has shown that IBM Hamiltonians derived from known EDFs in this region, produced eigenstates whose properties resemble those of SU(3)-PDS. The fact that these results are valid for both nonrelativistic and relativistic EDFs with several choices of pairing strengths, highlights the robustness of the PDS notion and its association with properties of the multi-nucleon dynamics in nuclei.

The results of the present investigation pave the way for a number of research avenues. (i) Exploring the microscopic origin of other types of PDSs, e.g., $\mathrm{SO}(6)-$ PDS in $\gamma$-soft nuclei. (ii) When a PDS is found to be manifested empirically in certain nuclei, it can be used to constrain, improve and optimize (e.g., choice of the pairing strength) a given EDF in that region. (iii) Exploiting the demonstrated linkage between the microscopic EDF framework and the algebraic PDS notion, to predict uncharted regions of exotic nuclei, awaiting to be explored, where partial symmetries can play a role.

\section{Acknowledgements}

The work of A.L. and N.G. is supported by the Israel Science Foundation. The work of K.N. is supported by the Tenure Track Pilot Programme of the Croatian Science Foundation and the École Polytechnique Fédérale de Lausanne, and the Project TTP-2018-07-3554 Exotic Nuclear Structure and Dynamics, with funds of the Croatian-Swiss Research Programme and also by the QuantiXLie Centre of Excellence, a project co-financed by the Croatian Government and European Union through the European Regional Development Fund - the Competitiveness and Cohesion Operational Programme (Grant KK.01.1.1.01.0004).

\section{References}

[1] F. Iachello (2015) "Lie Algebras and Applications". Springer-Verlag, Berlin.

[2] F. Iachello, A. Arima (1987) "The Interacting Boson Model". Cambridge University Press, Cambridge.

[3] J.N. Ginocchio, M.W. Kirson (1980) Phys. Rev. Lett. 441744.

[4] A.E.L. Dieperink, O. Scholten, F. Iachello (1980) Phys. Rev. Lett. 441747.

[5] A. Leviatan (2011) Prog. Part. Nucl. Phys. 6693.

[6] A. Leviatan (1996) Phys. Rev. Lett. 77818.

[7] A. Leviatan, I. Sinai (1999) Phys. Rev. C 60061301. 
Linking Partial Dynamical Symmetry to Nuclear Energy Density Functionals

[8] R.F. Casten, R.B. Cakirli, K. Blaum, A. Couture (2014) Phys. Rev. Lett. 113112501.

[9] A. Leviatan, J.E. García-Ramos, P. Van Isacker (2013) Phys. Rev. C 87 021302(R).

[10] J.E. García-Ramos, A. Leviatan, P. Van Isacker (2009) Phys. Rev. Lett. 102112502.

[11] P. Van Isacker, J. Jolie, T. Thomas, A. Leviatan (2015) Phys. Rev. C 92 011301(R).

[12] A. Leviatan (2007) Phys. Rev. Lett. 98242502.

[13] M. Macek, A. Leviatan (2014) Ann. Phys. (NY) 351302.

[14] A. Leviatan, D. Shapira (2016) Phys. Rev. C 93 051302(R).

[15] A. Leviatan, N. Gavrielov (2017) Phys. Scr. 92114005.

[16] A. Leviatan, N. Gavrielov, J.E. García-Ramos, P. Van Isacker (2018) Phys. Rev. C $98031302(\mathrm{R})$.

[17] K. Nomura, N. Gavrielov, A. Leviatan (2021) Phys. Rev. C 104, 044317.

[18] T. Dytrych, K.D. Launey, J.P. Draayer, D.J. Rowe, J.L. Wood, G. Rosensteel, C. Bahri, D. Langr, R.B. Baker (2020) Phys. Rev. Lett. 124042501.

[19] A.E. McCoy, M.A. Caprio, T. Dytrych, P.J. Fasano (2020) Phys. Rev. Lett. 125 102505.

[20] Y. Alhassid, A. Leviatan (1992) J. Phys. A 25 L1265.

[21] A. Leviatan (1987) Ann. Phys. (NY) 179201.

[22] M. Bender, P.-H. Heenen, P.-G. Reinhard (2003) Rev. Mod. Phys. 75121.

[23] L.M. Robledo, T.R. Rodríguez, R.R. Rodríguez-Guzmán (2019) J. Phys. G 46 013001.

[24] D. Vretenar, A.V. Afanasjev, G.A. Lalazissis, P. Ring (2005) Phys. Rep. 409101

[25] T. Nikšić, D. Vretenar, P. Ring (2011) Prog. Part. Nucl. Phys. 66519.

[26] P. Ring, P. Schuck (1980) “The Nuclear Many-Body Problem”. Springer, Berlin.

[27] A. Bohr, B.R. Mottelson (1975) "Nuclear Structure", Vol. II Benjamin, New York.

[28] P. Bonche, H. Flocard, P.-H. Heenen (2005) Comput. Phys. Commun. 17129.

[29] W. Ryssens, V. Hellemans, M. Bender, P.-H. Heenen (2015) Comput. Phys. Commun. 187175.

[30] E. Chabanat, P. Bonche, P. Haensel, J. Meyer, R. Schaeffer (1998) Nucl. Phys. A 635231.

[31] J. Dobaczewski, H. Flocard, J. Treiner (1984) Nucl. Phys. A 422103.

[32] T. Nikšić, N. Paar, D. Vretenar, P. Ring (2014) Comput. Phys. Commun. 1851808.

[33] T. Nikšić, D. Vretenar, P. Ring (2008) Phys. Rev. C 78034318.

[34] G.A. Lalazissis, T. Nikšić, D. Vretenar, P. Ring (2005) Phys. Rev. C 71024312.

[35] Y. Tian, Z.Y. Ma, P. Ring (2009) Phys. Lett. B 67644.

[36] K. Nomura, N. Shimizu, T. Otsuka (2008) Phys. Rev. Lett. 101142501.

[37] K. Nomura, N. Shimizu, T. Otsuka (2010) Phys. Rev. C 81044307.

[38] K. Nomura, T. Otsuka, N. Shimizu, L. Guo (2011) Phys. Rev. C 83 041302(R).

[39] J.-P. Delaroche, M. Girod, J. Libert, H. Goutte, S. Hilaire, S. Péru, N. Pillet, G.F. Bertsch (2010) Phys. Rev. C 81014303.

[40] Evaluated Nuclear Structure Data File (ENSDF) http://www.nndc.bnl.gov/ensdf. 